\title{
FREQUENCY-SPECIFIC PROJECTIONS OF INDIVIDUAL NEURONS IN CHICK BRAINSTEM AUDITORY NUCLEI ${ }^{1}$
}

\author{
STEVEN R. YOUNG AND EDWIN W RUBEL ${ }^{2}$ \\ Departments of Otolaryngology and Physiology, and Neuroscience Program, University of Virginia Medical Center, \\ Charlottesville, Virginia 22908
}

Received July 8, 1982; Revised January 17, 1983; Accepted January 18, 1983

\begin{abstract}
Nucleus magnocellularis and nucleus laminaris in the avian brainstem contain second- and thirdorder auditory neurons thought to be homologous to the mammalian anteroventral cochlear nucleus and medial superior olivary nucleus, respectively. Nucleus laminaris in the chicken is a tonotopically organized sheet of bipolar neurons; each of these neurons receives spatially segregated bilateral innervation from the two magnocellular nuclei. In the present study, this projection was studied at the single cell level by analyzing the pattern of terminal arborizations of individual horseradish peroxidase-filled axons. Reconstruction of the terminal arborizations of nucleus magnocellularis axons revealed that each axon forms an elongated band of endings, the long axis of which is parallel to the physiologically defined isofrequency bands. Within a band, the individual terminal collaterals form distinct patches separated by areas without endings. We suggest that the elongated terminal fields provide the basis of the tonotopic organization observed in nucleus laminaris and that the trajectories of the ipsilateral and contralateral axons may provide differential conduction delays that are important for binaural integration of acoustic information.
\end{abstract}

A great deal is known about patterns of connections between populations of neurons in vertebrate sensory and motor systems; yet, very little is known about the connectivity of individual cells within those patterns. Physiological and behavioral specificity could result from very specific point-to-point connections of cells in one group with those of another, or it could depend on a broader pattern of overlapping connections.

The brainstem auditory pathways of the chick offer a favorable preparation in which to study the role of individual axons in the formation of a topographically organized projection. Auditory information reaches the brain from the cochlea via the eighth cranial nerve. The avian eighth nerve forms the primary input to the ipsilateral nucleus magnocellularis (NM), a homogeneous group of

\footnotetext{
' We would like to thank Drs. Lincoln Gray, Dianne Durham, Oswald Steward, and John Wilson for helpful advice; Ms. Doris Hannum for technical help; and Mr. Werner Frewer for building some of our apparatus. Special thanks are due to Dr. William Lippe for extensive analytical help in understanding the relationship between the single cell terminal fields and the frequency axis of nucleus laminaris. This work was supported by National Institutes of Health Grant NS 15478, Research Career Development Award NS 00305, and funds from the Lions of Virginia Hearing Foundation.

${ }^{2}$ To whom correspondence should be addressed at Department of Otolaryngology, Box 430, University of Virginia Medical Center, Charlottesville, VA 22908.
}

cells in the dorsal medulla (Boord and Rasmussen, 1963; Ramon y Cajal, 1971; Parks and Rubel, 1978; Whitehead and Morest, 1981; Jhaveri and Morest, 1982). Each NM cell projects bilaterally onto the third-order neurons which are in nucleus laminaris. Nucleus laminaris (NL) of the chicken is composed of a monolayer sheet of neurons; each cell within the layer extends tufts of dendrites both dorsally and ventrally. 'The dorsal dendrites of NL cells receive input from the ipsilateral NM, whereas the ventral dendrites are innervated by axons from the contralateral NM (Boord, 1968; Parks and Rubel, 1975; Smith and Rubel, 1979). This organization is schematically illustrated in Figure 1.

There is a topographic projection from the cochlea onto NM and also from NM onto each NL (Parks and Rubel, 1975). The frequency organizations of both NM and NL have been described quantitatively (Rubel and Parks, 1975). Cells at progressively rostral and medial locations in each nucleus are most sensitive to successively higher frequencies. Thus, a line extending from the caudolateral to the rostromedial pole of NL describes the frequency axis of the nucleus. The NL cells on a line orthogonal to this axis form an isofrequency band.

This paper reports a study of axonal terminal fields of individual NM neurons and their relation to the functional organization of NL. The orientation of the frequency axis of NL provides a physiological reference with 


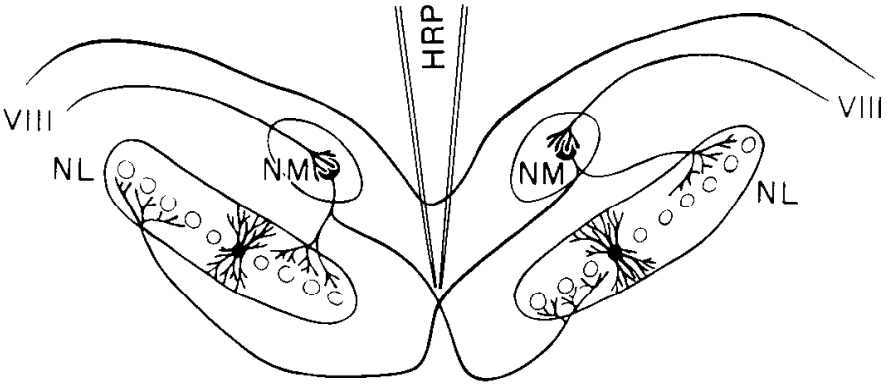

Figure 1. Schematic diagram of the major afferent and efferent connections of nucleus magnocellularis $(N M)$ showing the position of the injection pipette used in this study. Note the segregated dendritic regions of nucleus laminaris $(N L)$ and the calyceal endings (end bulbs of Held) of the auditory nerve (VIII). Adapted from Rubel et al. (1981).

which to compare the pattern of axonal terminals of the afferent NM cells.

\section{Materials and Methods}

We investigated the terminal arborizations of NM cells by using an in vitro brainstem preparation from 16- to 18-day chick embryos. After cutting the cranial nerves and the cerebellar peduncles, the isolated brainstem was removed from the chick and maintained under oxygenated avian Tyrode's solution ( $139 \mathrm{~mm} \mathrm{NaCl}, 3 \mathrm{~mm} \mathrm{KCl}$, $17 \mathrm{mM} \mathrm{NaHCO}_{3}, 1 \mathrm{~mm} \mathrm{MgCl}, 3 \mathrm{mmCaCl}, 2.2 \mathrm{gm} /$ liter of dextrose, equilibrated with $95 \% \mathrm{O}_{2} / 5 \% \mathrm{CO}_{2}$ ) at about $32^{\circ} \mathrm{C}$ for 3 to $4 \mathrm{hr}$. We have previously found that physiological responsiveness is usually maintained for more than $8 \mathrm{hr}$ under similar conditions (Hackett et al., 1982). Pipettes were filled with $2 \%$ horseradish peroxidase (HRP; Sigma, type VI), $0.2 \%$ fast green, and $0.2 \mathrm{M} \mathrm{NaCl}$ in $0.01 \mathrm{~m}$ Tris buffer, $\mathrm{pH}$ 7.6. The pipette tip was broken to an outside diameter of 2 to $5 \mu \mathrm{m}$. The NM cell axons were filled with HRP by placing the pipette tip, under visual control, into the midline at the decussation of the NM axons en route to the contralateral NL (Fig. 1). After cutting a small number of axons by a slight movement of the pipette tip, a small HRP injection was made by applying pressure pulses to the back of the pipette. Twoto 5 -msec pulses of $10 \mathrm{psi}$ were applied once every $1.1 \mathrm{sec}$ for 30 to $60 \mathrm{~min}$ using a General Valve Corporation "Picospritzer." An alternative method which sometimes produced good results was to make small pressure and/ or current injections of HRP while recording an extracellular unit response to eighth nerve stimulation from a cell body in NM (Hackett et al., 1982).

Following the HRP injection, the preparation was maintained for $2 \mathrm{hr}$ to allow for complete filling of the cell body and terminal arborization. The brainstem was then fixed by immersion in $3 \%$ glutaraldehyde, $3 \%$ sucrose in $0.1 \mathrm{M} \mathrm{PO}_{4}$ buffer $(\mathrm{pH} 7.3$ ) at room temperature for 30 min, followed by overnight equilibration in $30 \%$ sucrose in $\mathrm{PO}_{4}$ buffer with $3 \%$ glutaraldehyde. Fifty-micron frozen sections were cut in the coronal plane, mounted on subbed slides, and reacted with diaminobenzidine tetrahydrochloride using the cobalt/nickel intensification procedure of Adams (1981). Sections were then dehydrated and coverslipped without counterstaining. This technique yields a black reaction product against an other- wise unstained background. In the best preparations only one to four axons incorporated the HRP and were heavily stained down to their finest axonal terminals. In such preparations the individual axons can be followed throughout their course.

To reconstruct the complete axonal trajectory and terminal arborization, filled axons were traced in detail from serial sections at high magnification $(\times 1250)$ with the aid of a camera lucida. Composite drawings (Fig. 2) were made by retracing all of the axon segments of a given cell onto a single sheet of paper. Such composites provide a view of the cell as a whole in the coronal plane but collapse the anterior-posterior dimension. Plots of NL as if seen from above (horizontal planar projections, Fig. 4) were made by graphing the medial and lateral edges of the laminar nucleus from each section as a function of the anterior-posterior position of the section. Terminal fields were plotted on these NL outlines as clusters of terminals. If clusters of terminals were not clearly distinct within a large group of terminals, the group was arbitrarily divided into clusters of a size comparable to that of clusters that were distinct. The same method of planar projection has been used previously to map the frequency organization of NM and NL and the dendritic organization of NL (Rubel and Parks, 1975; Smith and Rubel, 1979; Smith, 1981).

The terminal arbors of NM neurons were also investigated in adult chickens using an in vivo preparation. In three experiments 15 - to 20 -day posthatch chickens were used. The surgical and preparative procedures were essentially similar to those used in earlier studies (Rubel and Parks, 1975). The fourth ventricle was exposed, and HRP injections were made using the same procedure described above. Following HRP injection the cavity was packed with Gelfoam and the skin was closed with cyanoacrylic glue. The chicken was then allowed to survive 12 to $18 \mathrm{hr}$ before being deeply anesthetized and perfused transcardially with the fixative noted above. The brain was removed and processed as described above.

\section{Results}

Six terminal fields of NM cells from two 17-day embryos were chosen for complete reconstruction on the basis of dark, and apparently complete, filling of terminals. These axons and their collateral branches could be readily distinguished from the terminal fields of other stained fibers. The NM cell bodies and their terminal arborizations in NL extended over six to eight $50-\mu \mathrm{m}$ sections in each of these cases.

Each NM cell sends a single axon ventromedially to the point of its first bifurcation just below NM. This first bifurcation gives rise to an ipsilaterally and a contralaterally bound axonal branch. The ipsilateral branch reflects dorsolaterally and somewhat anteriorly to course back through or above the rest of NM (Fig. 2). Examination of cells stained by intracellular HRP injections or with the Golgi method have revealed a similar course (Hackett et al., 1982; Jhaveri and Morest, 1982). This branch continues beneath the ventricular surface to the lateral edge of NM, where it turns ventrally and then begins ramifying to form terminal arborizations in the 


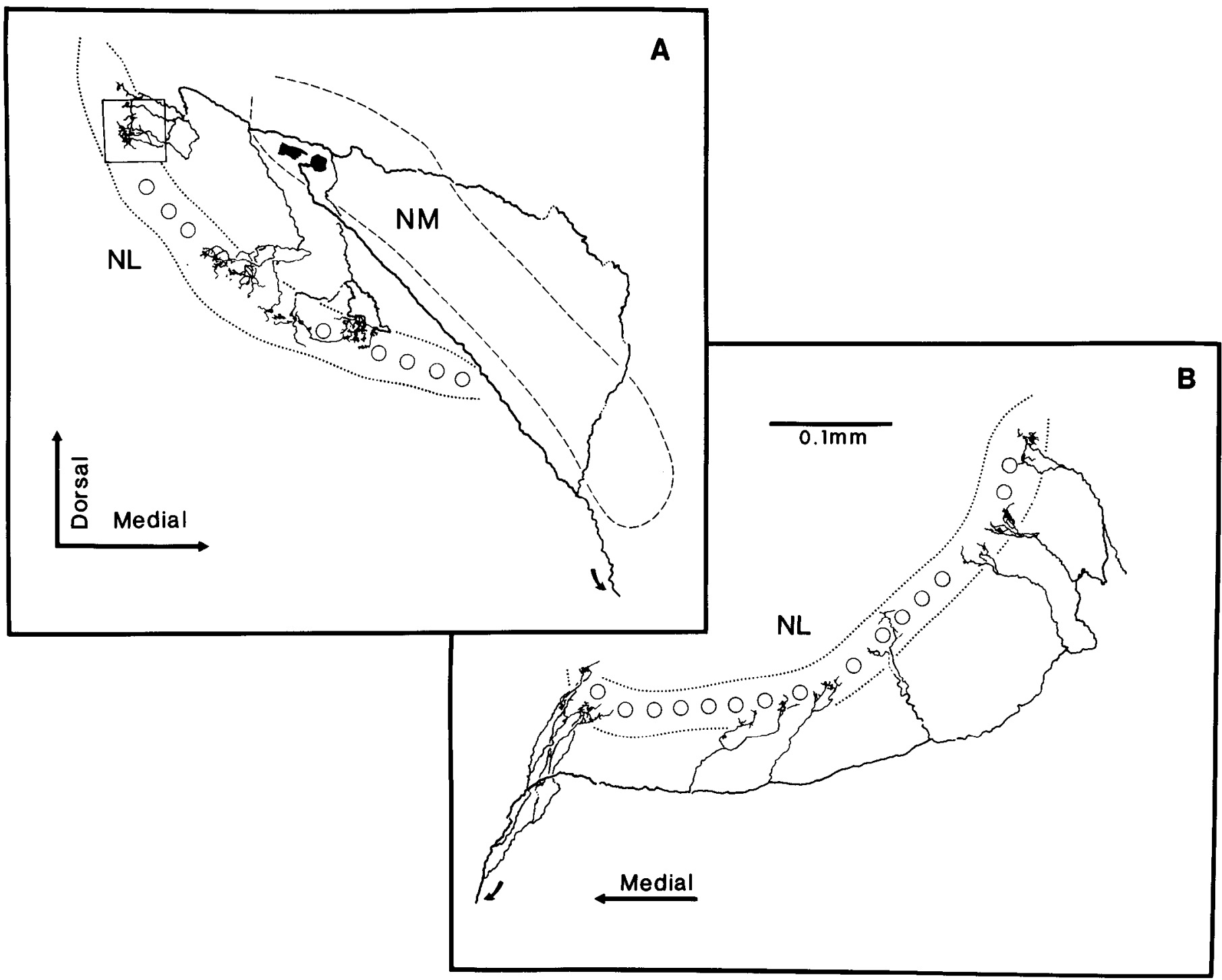

Figure 2. A, Composite camera lucida drawing of one $\mathrm{NM}$ cell and its ipsilateral axonal arborization superimposed on a single cross-sectional drawing of NL. The HRP-labeled cell body is indicated by the pointer in the upper center of the figure. NM and NL are indicated schematically by the dashed and dotted lines, respectively. The primary axon can be seen leaving its cell body and heading in a ventromedial direction. It passes quite close to NL cells (on which it will eventually terminate) without branching or forming terminals. The first branch point is in the lower right of the figure. The contralaterally bound branch is indicated by the arrow. It curves medially and crosses the midline under the floor of the fourth ventricle. The ipsilateral branch turns dorsad and reflects back through or above NM to course anterolaterally beneath the floor of the fourth ventricle. Its branches, which drop ventrally through or lateral to NM and appear to cross the primary axon on their way to NL, are actually located several sections anterior to the main axon. Note the distribution of the terminal arborization into patches. The boxed area in the upper left is shown at high magnification in Figure 3. The horizontal planar projection of this cell is shown in Figure $4 C$. Scale is the same as $B$. $B$, Composite drawing of the contralateral terminal field of an NM cell. The NL cell body layer and neuropil outline are shown schematically. This terminal field is plotted in Figure $4 E$. Bar, $100 \mu \mathrm{m}$.

dorsal neuropil and the cell body layer of the ipsilateral NL.

The contralateral branch passes ventrally in the dorsal cochlear tract, crosses the midline, and sweeps dorsally to the medial end of the contralateral NL where it begins to branch. This collateral then courses further anterolaterally, through the area just ventral to NL, and sends branches into the ventral neuropil and cell body layer of the contralateral NL.

NM axons terminate in a series of clusters of grapelike boutons in the neuropil and cell body layer of NL. Both terminal boutons and boutons en passant dot the extremely fine terminal fibers. Often the terminal fibers and boutons of a given axonal branch form a cluster distinct from other clusters of terminals. Figure 3 shows a terminal cluster which overlaps with two others but which is in a different focal plane.

The axons giving rise to terminal fields on the dorsal side of NL could usually be traced to their parent cell body in the ipsilateral NM. In most cases terminal fields in the ventral neuropil could not be traced to their contralateral cell bodies because their axons were cut by the injection pipette and the cut ends had become separated. In no case, however, have we observed more than 


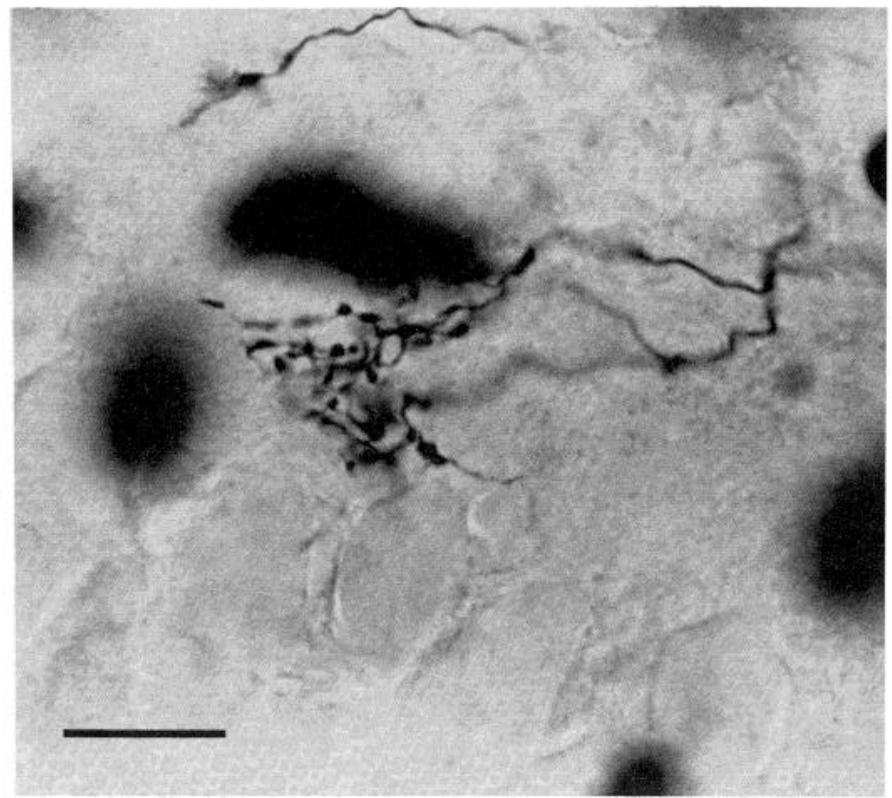

Figure 3. Photomicrograph of a cluster of boutons in the dorsal NL neuropil of a 17-day chick embryo. Drawn in Figure $2 A$ and plotted in Figure $4 C$, this cluster overlaps with two others that lie in a different focal plane. The large unstained cells in the lower part of the figure are the cell bodies of NL neurons. The dorsal NL neuropil is the relatively lightly textured area which occupies most of the center of the figure. The large, out of focus dark masses are red blood cells. $\times 100$ planapochromatic objective, numerical aperture $=1.3 . \mathrm{Bar}, 10$ $\mu \mathrm{m}$.

one axon from any one NM cell crossing the midline. It was, therefore, concluded that those axonal branches that could be confidently traced to a single axon at the midline constituted the whole contralateral projection of a single NM cell.

Two features of the terminal fields of both the ipsilateral and the contralateral branches became apparent when NM axons were followed through successive sections. First, the terminal fields showed a definite orientation. In each coronal section the terminals of one NM axon covered only a small part of the medial-lateral extent of NL; the area of NL which it covered shifted systematically from the medial edge in relatively posterior sections to progressively lateral areas in more anterior sections.

In order to examine the orientation of the terminal fields more precisely, the area of NL covered by the terminals of each of the six NM axons was plotted on a horizontal planar projection of the outline of the respective NL. The positions of clusters of terminal arbors from each axon are shown in Figure 4. Also shown is the approximate orientation of the tonotopic organization of NL as plotted from microelectrode mapping data of Rubel and Parks (1975). (The orientation of the tonotopic map in 17-day embryos matches that of the hatchling (Lippe and Rubel, 1983).) It is strikingly apparent from Figure 4 that the NM terminal fields form narrow bands orthogonal to the frequency axis of NL. Each terminal field extends across virtually the entire width of the nucleus orthogonal to the tonotopic axis (i.e., along an isofrequency band). This is a distance of about $450 \mu \mathrm{m}$. On the other hand, the width of the fields in their short dimension, from rostromedial to caudolateral (along the frequency axis) never exceeds 50 to $75 \mu \mathrm{m}$. Thus, a single NM cell that appears to innervate the entire extent of $\mathrm{NL}$ along an isofrequency band projects across only about $5 \%$ of the nucleus along the tonotopic dimension of NL.

The second feature of the NM axon terminal fields that was apparent from examination of serial sections was the nonuniform distribution of terminals from each axon. There was a segregation of clusters of terminals into patches which were separated from one another by clear areas of roughly comparable size. This division of the NM terminal fields into patches is apparent in the examples shown in Figure 2. In each of the six terminal fields that were completely reconstructed, the clusters of terminal arbors formed three distinct patches. Rarely, as in Figure 2A, a small caliber fiber could be seen crossing from one patch of terminals to another. More often the terminals of any major axon branch all occurred within the same patch.

Many of the more than eighty 16- to 18-day embryos used in developing the injection procedures showed staining that was not suitable for reconstruction of individual cells, most often because more than three or four axons were stained. Nevertheless, in many of these cases the relatively small number of axons filled (less than a dozen) allowed the qualitative characteristics of the terminal fields to be discerned without detailed reconstructions. In these cases also the terminal fields were elongated orthogonal to the frequency axis and were divided into three patches. The results from three 15- to 20-day posthatch chickens in which axons were stained in vivo with HRP injections also confirm this pattern. Finally, recent results in our laboratory using a cobalt/lysine complex with a similar in vivo injection procedure reveal the same projection pattern in chickens 3 to 4 weeks after hatching (J. A. Wilson, S. R. Young, and E. W Rubel, unpublished observations).

\section{Discussion}

This study describes the shape and orientation of the terminal fields in nucleus laminaris of axons from nucleus magnocellularis. The orientation of these fields corresponds to that of the physiologically defined isofrequency bands (Rubel and Parks, 1975). The tonotopic organization of $\mathrm{NL}$ is, therefore, the result of the orderly arrangement of these oriented terminal fields.

Other investigators have also examined terminal field patterns of individual cells in an attempt to understand the way information is processed in a given region of the CNS. Scheibel and Scheibel (1974) described the threedimensional structure of the terminal fields in the medial superior olivary nucleus, the likely mammalian homologue of NL. They found a longitudinal arborization of afferents from the ventral cochlear nuclei that seemed to form sheets of neuropil orthogonal to the tonotopic organization described in physiological studies (Tsuchitani and Boudreau, 1966; Guinan et al., 1972). Although the complexity of the mammalian superior olivary complex 


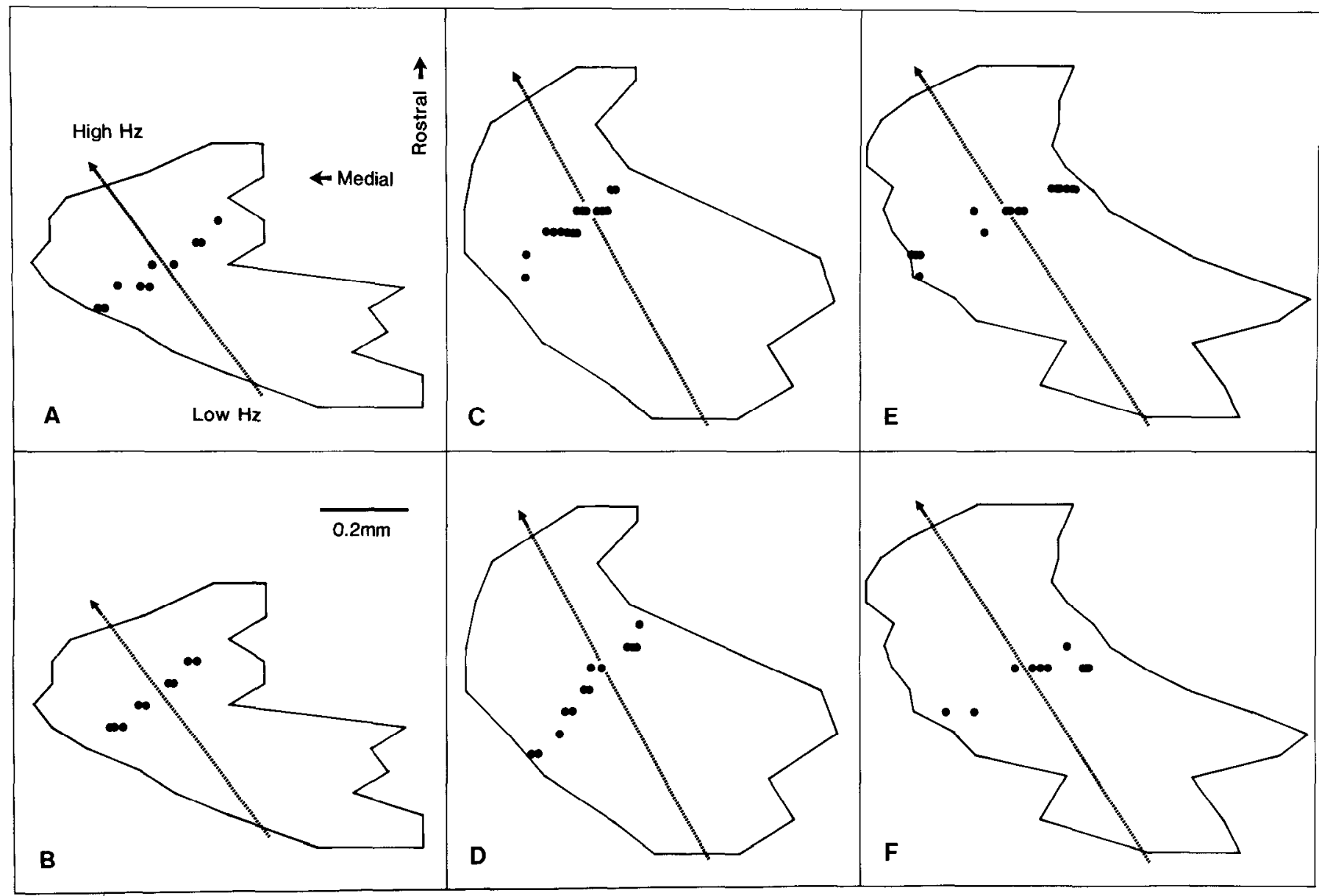

Figure 4. Horizontal planar projections of the terminal fields of six NM axons in NL. The axis of the tonotopic organization in $\mathrm{NL}$ is indicated approximately by the dashed line and arrow on each projection. It runs from caudolateral (low frequencies) to rostromedial (high frequencies) in each case. $A, B, D$, and $E$ are plots of ventral terminal fields; $C$ and $F$ show terminals in the dorsal neuropil. Each circle represents a cluster of terminals similar to that shown in Figure 3 . The diameter of the circles approximates the average diameter of a cluster. Cluster sizes range around this average by a factor of about $\times 2$. Anterior-posterior positions are plotted in 50- $\mu \mathrm{m}$ intervals. As can be appreciated from the coronal views of NL shown in Figure 2 , the curvature of the lateral portion of NL masks the discontinuities of the terminal fields when these fields are viewed in the horizontal plane.

makes their data difficult to interpret, their findings are similar to what we have described in the chick.

Terminal fields of individual cells have also been studied at many levels of the visual system. Intracellular labeling has shown that physiologically defined X- and Y-type cells have distinct morphological characteristics, in both their axonal and their dendritic fields (Ferster and LeVay, 1978; Gilbert and Wiesel, 1979; Bowling and Michael, 1980; Friedlander et. al., 1981). Gilbert and Wiesel (1979), in addition, have been able to relate indirectly, patterns of aiferent axonal termination to receptive field characteristics of visual cortex neurons. These authors found that cells in striate cortex with "simple" receptive fields occur in the region of termination of the optic radiation and project, in turn, to layers of the cortex where they are likely to synapse on "complex" cells. Their results support a proposed serial processing of visual information by the various cell types of the visual cortex.

Beyond maintaining the topography of the basilar papilla in the projection from NM to NL, any assertions regarding the functional significance of the projection pattern described here must be highly speculative. However, differences between the ipsilateral and the contralateral pattern deserve some consideration in relation to the proposed role of this system in binaural information processing (Parks and Rubel, 1975; Jenkins and Masterton, 1979). Theoretical models have been advanced which describe some of the aspects of sound localization in human subjects in terms of a hypothetical array of cells, each cell in the array receiving binaural inputs with characteristic differences in transmission times from the two ears (Jeffress, 1948; Colburn, 1977). It has been suggested that NL might be the site of such binaural interaction in birds (Parks and Rubel, 1975, p. 446). These authors proposed that if each NM axon provided a unique conduction delay, then those NL cells whose inputs provided binaural delays which precisely compensated the difference in the arrival times of a sound at the ears would be the cells most activated by that sound. If the NM terminal arborizations were restricted to a small part of NL, then the NL cells might be functionally organized along two dimensions, one corresponding to frequency and the other to location of a sound source. 
The present finding, that the terminal fields in NL are spread across the nucleus, is inconsistent with this simple model. The pattern illustrated in Figure 2 does, however, suggest an alternative model for producing an appropriate delay. The length of the axon from the NM cell body to each of the patches of terminals in the ipsilateral dorsal NL neuropil appears to be roughly equal. In contrast, the length of the axon from a cell in NM to its terminals in the contralateral NL is progressively longer from the medial to the lateral patches. This allows the possibility that characteristic signal delays might be represented by different signal path lengths within the contralateral projections of individual NM cells.

\section{References}

Adams, J. C. (1981) Heavy metal intensification of DAB-based HRP reaction product. J. Histochem. Cytochem. 29: 775.

Boord, R. L. (1968) Ascending projections of the primary cochlear nuclei and nucleus laminaris in the pigeon. J. Comp. Neurol. 133: 523-542.

Boord, R. L., and G. L. Rasmussen (1963) Projection of the cochlear and lagenar nerves on the cochlear nuclei of the pigeon. J. Comp. Neurol. 120: 463-475.

Bowling, D. B., and C. R. Michael (1980) Projection patterns of single physiologically characterized optic tract fibres in cat. Nature 286: 899-902.

Colburn, H. S. (1977) Theory of binaural interaction based on auditory-nerve data. II. Detection of tones in noise. J. Acoust. Soc. Am. 61: 525-533.

Ferster, D., and S. LeVay (1978) The axonal arborizations of lateral geniculate neurons in the striate cortex of the cat. J. Comp. Neurol. 182: 923-944.

Friedlander, M. J., C. -S. Lin, L. R. Stanford, and S. M. Sherman (1981) Morphology of functionally identified neurons in lateral geniculate nucleus of the cat. J. Neurophysiol. 46: $80-129$.

Gilbert, C. D., and T. N. Wiesel (1979) Morphology and intracortical projections of functionally characterized neurons in the cat visual cortex. Nature 280: 120-125.

Guinan, J. J., B. E. Norris, and S. S. Guinan (1972) Single auditory units in the superior olivary complex. II. Locations of unit categories and tonotopic organization. Int. J. Neurosci. 4: $147-166$.

Hackett, J. T., H. Jackson, and E. W Rubel (1982) Synaptic excitation of the second and third order auditory neurons in the avian brain stem. Neuroscience 7: 1455-69.

Jeffress, L. A. (1948) A place theory of sound localization. J. Comp. Physiol. Psychol. 41: 35-39.

Jenkins, W. M., and R. B. Masterton (1979) Sound localization in pigeon (Columba livia). J. Comp. Physiol. Psychol. 93: 403-413.

Jhaveri, S., and D. K. Morest (1982) Neuronal architecture in nucleus magnocellularis of the chicken auditory system with observations on nucleus laminaris: A light and electron microscope study. Neuroscience 7: 809-836.

Lippe, W. R., and E. W Rubel (1983) Development of the place code: Tonotopic organization. Science 219: 514-516.

Parks, T. N., and E. W Rubel (1975) Organization of projections from n. magnocellularis to $n$. laminaris. J. Comp. Neurol. 164: $435-448$.

Parks, T. N., and E. W Rubel (1978) Organization and development of the brainstem auditory nuclei of the chicken: Primary afferent projections. J. Comp. Neurol. 180: 439-448.

Ramon y Cajal, S. (1971) The acoustic nerve: Its cochlear branch or cochlear nerve. Translated from Histologie $d u$ Systeme Nerveux de l'Homme et des Vertebres, Tome I, pp. 774-838, 1952. National Technical Information Service Publication No. PB-205 473, Washington, D.C.

Rubel, E. W, and T. N. Parks (1975) Organization and development of brain stem auditory nuclei of the chicken: Tonotopic organization of $\mathbf{n}$. magnocellularis and $\mathrm{n}$. laminaris. $\mathrm{J}$. Comp. Neurol. 164: 411-434.

Rubel, E. W, Z. D. J. Smith, and O. Steward (1981) Sprouting in the avian brainstem auditory pathway: Dependence on dendritic integrity. J. Comp. Neurol. 202: 397-414.

Scheibel, M. E., and A. B. Scheibel (1974) Neuropil organization in the superior olive of the cat. Exp. Neurol. 43: 339-348.

Smith, Z. D. J. (1981) Organization and development of brain stem auditory nuclei of the chicken: Dendritic development in n. laminaris. J. Comp. Neurol. 203: 309-333.

Smith, D. J., and E. W Rubel (1979) Organization and development of brain stem auditory nuclei of the chicken: Dendritic gradients in nucleus laminaris. J. Comp. Neurol. 186: 213240.

Tsuchitani, C., and J. C. Boudreau (1966) Single unit analysis of cat superior olive S segment with tonal stimuli. J. Neurophysiol. 29: 684-697.

Whitehead, M. C., and D. K. Morest (1981) Dual populations of efferent and afferent cochlear axons in the chicken. Neuroscience 6: 2351-2365. 